\title{
Intersection local times of fractional Brownian motions with $H \in(0,1)$ as generalized white noise functionals
}

\author{
Custódia Drumond \\ DME, University of Madeira, P 9000-390 Funchal, Portugal \\ CCM, University of Madeira, P 9000-390 Funchal, Portugal \\ collie@uma.pt \\ Maria João Oliveira \\ Universidade Aberta, P 1269-001 Lisbon, Portugal \\ CMAF, University of Lisbon, P 1649-003 Lisbon, Portugal \\ oliveira@cii.fc.ul.pt \\ José Luís da Silva \\ DME, University of Madeira, P 9000-390 Funchal, Portugal \\ CCM, University of Madeira, P 9000-390 Funchal, Portugal \\ luis@uma.pt
}

\begin{abstract}
In $\mathbb{R}^{d}$, for any dimension $d \geq 1$, expansions of self-intersection local times of fractional Brownian motions with arbitrary Hurst coefficients in $(0,1)$ are presented. The expansions are in terms of Wick powers of white noises (corresponding to multiple Wiener integrals), being well-defined in the sense of generalized white noise functionals.
\end{abstract}

Dedicated to Professor Ludwig Streit on the occasion of his $70^{\text {th }}$ Birthday

Keywords: Fractional Brownian motion; Fractional white noise analysis; Local time

2000 AMS Classification: 60H40, 60G15, 28C20, 46F 25 


\section{Introduction}

Intersection local times of fractional Brownian motion (fBm) has been studied by many authors, see e.g. the works done by Gradinaru et al. GRV03, Nualart et al. [HN07], [HN05], Rosen [Ros87], and the references therein.

We may consider intersections of simple paths of a $\mathrm{fBm}$ with themselves or with other independent fractional Brownian motion as in [NOL07. Within the white noise analysis framework, we concentrate here in the simple selfintersection problem using a similar approach to Faria et al. dFHSW97, dFDS00 to study the case $H=1 / 2$ (the classical Brownian motion). This approach has the advantage that the underlying probability space does not depend on the Hurst coefficient under consideration. As a consequence, we analyze the self-intersections of a fBm, with Hurst coefficient being any possible value in $(0,1)$. As one may expect, independently of the Hurst coefficient under consideration, self-intersections become scarce as the dimension $d$ increases.

An informal but suggestive definition of self-intersection local time of a generic $d$-dimensional $\mathrm{fBm} B$ is in terms of an integral over Donsker's $\delta$ function

$$
L=\int d^{2} t \delta\left(B\left(t_{2}\right)-B\left(t_{1}\right)\right)
$$

intended to measure the amount of "time" the process spends intersecting itself.

A rigorous definition, such as, e.g., through a sequence of Gaussians approximating the $\delta$-function,

$$
(2 \pi \varepsilon)^{-d / 2} \exp \left(-\frac{|x|^{2}}{2 \varepsilon}\right), \quad \varepsilon>0,
$$

will make $L$ increasingly singular, and various "renormalizations" have to be done as the dimension $d$ increases. For $d>1$ and $1 / d \leq H<3 /(2+d)$, the expectation diverges in the limit and must be subtracted. The $L^{2}$-properties related to this situation have been analyzed in [HN05]. For other $H$ values further kernel terms must be subtracted (Theorem 7 below).

In this work we are particularly interested in the chaos decomposition of $L$. We expand $L$ in terms of Wick powers [HKPS93] of white noise, an expansion which corresponds to that in terms of multiple Wiener integrals when one considers the Wiener process as the fundamental random variable. This allows us to derive the kernels for $L$. Due to the local structure of the 
Wick powers, the kernel functions are relatively simple and exhibit clearly the dimension dependence singularities of $L$ (Proposition 8). For comparison, we also calculate the regularized kernel functions corresponding to the Gaussian $\delta$-sequence mentioned above.

The paper is organized as follows. In Section 2 we review the necessary background of white noise analysis and in Section 3 we present the main results of this work and their proofs. We shall mention that after the conclusion of our work we found the recent paper [Rez07] with related results.

\section{Gaussian white noise calculus}

In this section we briefly recall the concepts and results of white noise analysis used throughout this work (for a detailed explanation see e.g. [BK88], [Hid75], [HKPS93], HØUZ96], Kon75, KT80a], KT80b], [Kuo96], Oba94]).

\subsection{Fractional Brownian motion}

The starting point of white noise analysis is the real Gelfand triple

$$
S_{d}(\mathbb{R}) \subset L_{d}^{2}(\mathbb{R}) \subset S_{d}^{\prime}(\mathbb{R}),
$$

where $L_{d}^{2}(\mathbb{R}):=L^{2}\left(\mathbb{R}, \mathbb{R}^{d}\right), d \geq 1$, is the real Hilbert space of all vector valued square integrable functions with respect to the Lebesgue measure on $\mathbb{R}, S_{d}(\mathbb{R})$ and $S_{d}^{\prime}(\mathbb{R})$ are the Schwartz spaces of the vector valued test functions and tempered distributions, respectively. We shall denote the $L_{d}^{2}(\mathbb{R})$-norm by $|\cdot|$ and the dual pairing between $S_{d}^{\prime}(\mathbb{R})$ and $S_{d}(\mathbb{R})$ by $\langle\cdot, \cdot\rangle$, which is defined as the bilinear extension of the inner product on $L_{d}^{2}(\mathbb{R})$, i.e.,

$$
\langle\mathbf{g}, \mathbf{f}\rangle=\sum_{i=1}^{d} \int_{\mathbb{R}} d x g_{i}(x) f_{i}(x)
$$

for all $\mathbf{g}=\left(g_{1}, \ldots, g_{d}\right) \in L_{d}^{2}(\mathbb{R})$ and all $\mathbf{f}=\left(f_{1}, \ldots, f_{d}\right) \in S_{d}(\mathbb{R})$. By the Minlos theorem, there is a unique probability measure $\mu$ on the $\sigma$-algebra $\mathcal{B}$ generated by the cylinder sets on $S_{d}^{\prime}(\mathbb{R})$ with characteristic function given by

$$
C(\mathbf{f}):=\int_{S_{d}^{\prime}(\mathbb{R})} d \mu(\omega) e^{i\langle\omega, \mathbf{f}\rangle}=e^{-\frac{1}{2}|\mathbf{f}|^{2}}, \quad \mathbf{f} \in S_{d}(\mathbb{R}) .
$$


In this way we have defined the white noise measure space $\left(S_{d}^{\prime}(\mathbb{R}), \mathcal{B}, \mu\right)$. Within this formalism, a version of the $d$-dimensional Wiener Brownian motion is given by

$$
\mathbf{B}(t):=\left(\left\langle\omega_{1}, \mathbb{1}_{[0, t]}\right\rangle, \ldots,\left\langle\omega_{d}, \mathbb{1}_{[0, t]}\right\rangle\right), \quad\left(\omega_{1}, \ldots, \omega_{d}\right) \in S_{d}^{\prime}(\mathbb{R})
$$

where $\mathbb{1}_{A}$ denotes the indicator function of a set $A$. For an arbitrary Hurst parameter $0<H<1, H \neq \frac{1}{2}$, a version of a $d$-dimensional fractional Brownian motion is given by

$$
\mathbf{B}_{H}(t):=\left(\left\langle\omega_{1}, M_{H} \mathbb{1}_{[0, t]}\right\rangle, \ldots,\left\langle\omega_{d}, M_{H} \mathbb{1}_{[0, t]}\right\rangle\right), \quad\left(\omega_{1}, \ldots, \omega_{d}\right) \in S_{d}^{\prime}(\mathbb{R}),
$$

where, for a generic real valued function $f$, and for $\frac{1}{2}<H<1$,

$$
\left(M_{H} f\right)(x):=\frac{K_{H}}{\Gamma\left(H-\frac{1}{2}\right)} \int_{x}^{\infty} d y f(y)(y-x)^{H-\frac{3}{2}}
$$

provided the integral exists for all $x \in \mathbb{R}$, while for $0<H<\frac{1}{2}$,

$$
\left(M_{H} f\right)(x):=\frac{\left(\frac{1}{2}-H\right) K_{H}}{\Gamma\left(H+\frac{1}{2}\right)} \lim _{\varepsilon \rightarrow 0^{+}} \int_{\varepsilon}^{\infty} d y \frac{f(x)-f(x+y)}{y^{\frac{3}{2}-H}},
$$

provided the limit exists for almost all $x \in \mathbb{R}$ (for more details see e.g. Ben03] and [PT00] and the references therein). Independently of the case under consideration, the normalizing constant $K_{H}$ is given by

$$
K_{H}=\Gamma\left(H+\frac{1}{2}\right)\left(\frac{1}{2 H}+\int_{0}^{\infty} d s\left((1+s)^{H-\frac{1}{2}}-s^{H-\frac{1}{2}}\right)\right)^{-\frac{1}{2}} .
$$

Apart from the operators (11), (2), we shall also consider the operator defined for $\frac{1}{2}<H<1$ by

$$
\left(M_{H}^{+} f\right)(x):=\frac{K_{H}}{\Gamma\left(H-\frac{1}{2}\right)} \int_{-\infty}^{x} d y f(y)(x-y)^{H-\frac{3}{2}}
$$

provided the integral exists for all $x \in \mathbb{R}$, and the operator defined for $0<$ $H<\frac{1}{2}$ by

$$
\left(M_{H}^{+} f\right)(x):=\frac{\left(\frac{1}{2}-H\right) K_{H}}{\Gamma\left(H+\frac{1}{2}\right)} \lim _{\varepsilon \rightarrow 0^{+}} \int_{\varepsilon}^{\infty} d y \frac{f(x)-f(x-y)}{y^{\frac{3}{2}-H}},
$$


provided the limit exists for almost all $x \in \mathbb{R}$.

There are several examples of functions $f$ for which $M_{H} f$ and $M_{H}^{+} f$ exist for any $H \in(0,1)$. For instance, $f=\mathbb{1}_{[0, t]}, t>0$, or $f \in S_{1}(\mathbb{R})$. For functions $f_{1}, f_{2}$ being either one of these two types it is easy to prove the following equality

$$
\int_{\mathbb{R}} d x f_{1}(x)\left(M_{H} f_{2}\right)(x)=\int_{\mathbb{R}} d x\left(M_{H}^{+} f_{1}\right)(x) f_{2}(x),
$$

showing that $M_{H}$ and $M_{H}^{+}$are dual operators. For more details and proofs see e.g. Ben03] and the references therein.

\subsection{Hida distributions and characterization results}

Let us now consider the complex Hilbert space $L^{2}\left(S_{d}^{\prime}(\mathbb{R}), \mathcal{B}, \mu\right)$. This space is canonically isomorphic to the symmetric Fock space of symmetric square integrable functions,

$$
L^{2}\left(S_{d}^{\prime}(\mathbb{R}), \mathcal{B}, \mu\right) \simeq\left(\bigoplus_{k=0}^{\infty} \operatorname{Sym} L^{2}\left(\mathbb{R}^{k}, k ! d^{k} x\right)\right)^{\otimes d}
$$

leading to the chaos expansion of the elements in $L^{2}\left(S_{d}^{\prime}(\mathbb{R}), \mathcal{B}, \mu\right)$,

$$
F\left(\omega_{1}, \ldots, \omega_{d}\right)=\sum_{\left(n_{1}, \ldots, n_{d}\right) \in \mathbb{N}^{d}}\left\langle: \omega_{1}^{\otimes n_{1}}: \otimes \cdots \otimes: \omega_{d}^{\otimes n_{d}}:, \mathbf{f}_{\left(n_{1}, \ldots, n_{d}\right)}\right\rangle
$$

with kernel functions $\mathbf{f}_{\left(n_{1}, \ldots, n_{d}\right)}$ in the Fock space. For simplicity, in the sequel we shall use the notation

$$
\mathbf{n}=\left(n_{1}, \cdots, n_{d}\right) \in \mathbb{N}^{d}, \quad n=\sum_{i=1}^{d} n_{i}, \quad \mathbf{n} !=\prod_{i=1}^{d} n_{i} !
$$

which reduces expansion (5) to

$$
F(\omega)=\sum_{\mathbf{n} \in \mathbb{N}^{d}}\left\langle: \omega^{\otimes \mathbf{n}}:, \mathbf{f}_{\mathbf{n}}\right\rangle, \quad \omega \in S_{d}^{\prime}(\mathbb{R}) .
$$

To proceed further we have to consider a Gelfand triple around the space $L^{2}\left(S_{d}^{\prime}(\mathbb{R}), \mathcal{B}, \mu\right)$. We will use the space $(S)^{*}$ of Hida distributions (or generalized Brownian functionals) and the corresponding Gelfand triple $(S) \subset L^{2}\left(S_{d}^{\prime}(\mathbb{R}), \mathcal{B}, \mu\right) \subset(S)^{*}$. Here $(S)$ is the space of white noise test 
functions such that its dual space (with respect to $L^{2}\left(S_{d}^{\prime}(\mathbb{R}), \mathcal{B}, \mu\right)$ ) is the space $(S)^{*}$. Instead of reproducing the explicit construction of $(S)^{*}$ (see e.g. [HKPS93]), in Theorem 2 below we define this space by its $S$-transform. We recall that given a $\mathbf{f} \in S_{d}(\mathbb{R})$, and the Wick exponential

$$
: \exp (\langle\omega, \mathbf{f}\rangle)::=\sum_{\mathbf{n} \in \mathbb{N}^{d}} \frac{1}{\mathbf{n} !}\left\langle: \omega^{\otimes \mathbf{n}}:, \mathbf{f}^{\otimes \mathbf{n}}\right\rangle=C(\mathbf{f}) e^{\langle\omega, \mathbf{f}\rangle}
$$

we define the $S$-transform of a $\Phi \in(S)^{*}$ by

$$
S \Phi(\mathbf{f}):=\langle\langle\Phi,: \exp (\langle\cdot, \mathbf{f}\rangle):\rangle\rangle, \quad \forall \mathbf{f} \in S_{d}(\mathbb{R}) .
$$

Here $\langle\langle\cdot, \cdot\rangle\rangle$ denotes the dual pairing between $(S)^{*}$ and $(S)$ which is defined as the bilinear extension of the sesquilinear inner product on $L^{2}\left(S_{d}^{\prime}(\mathbb{R}), \mathcal{B}, \mu\right)$. We observe that the multilinear expansion of (6),

$$
S \Phi(\mathbf{f}):=\sum_{\mathbf{n}}\left\langle F_{\mathbf{n}}, \mathbf{f}^{\otimes \mathbf{n}}\right\rangle
$$

extends the chaos expansion to $\Phi \in(S)^{*}$ with distribution valued kernels $F_{\mathbf{n}}$ such that

$$
\langle\langle\Phi, \varphi\rangle\rangle=\sum_{\mathbf{n}} \mathbf{n} !\left\langle F_{\mathbf{n}}, \varphi_{\mathbf{n}}\right\rangle
$$

for every generalized test function $\varphi \in(S)$ with kernel functions $\varphi_{\mathbf{n}}$.

In order to characterize the space $(S)^{*}$ through its $S$-transform we need the following definition.

Definition 1 A function $F: S_{d}(\mathbb{R}) \rightarrow \mathbb{C}$ is called a $U$-functional whenever 1. for every $\mathbf{f}_{1}, \mathbf{f}_{2} \in S_{d}(\mathbb{R})$ the mapping $\mathbb{R} \ni \lambda \longmapsto F\left(\lambda \mathbf{f}_{1}+\mathbf{f}_{2}\right)$ has an entire extension to $\lambda \in \mathbb{C}$,

2. there are constants $K_{1}, K_{2}>0$ such that

$$
|F(z \mathbf{f})| \leq K_{1} \exp \left(K_{2}|z|^{2}\|\mathbf{f}\|^{2}\right), \quad \forall z \in \mathbb{C}, \mathbf{f} \in S_{d}(\mathbb{R})
$$

for some continuous norm $\|\cdot\|$ on $S_{d}(\mathbb{R})$.

We are now ready to state the aforementioned characterization result.

Theorem 2 ([KLP+96], [PS91]) The S-transform defines a bijection between the space $(S)^{*}$ and the space of $U$-functionals. 
As a consequence of Theorem 2 one may derive the next two statements. The first one concerns the convergence of sequences of Hida distributions and the second one the Bochner integration of families of the same type of distributions (for more details and proofs see e.g. [HKPS93], [KLP ${ }^{+96}$ ], [PS91]).

Corollary 3 Let $\left(\Phi_{n}\right)_{n \in \mathbb{N}}$ be a sequence in $(S)^{*}$ such that

(i) For all $\mathbf{f} \in S_{d}(\mathbb{R}),\left(\left(S \Phi_{n}\right)(\mathbf{f})\right)_{n \in \mathbb{N}}$ is a Cauchy sequence in $\mathbb{C}$,

(ii) There are $K_{1}, K_{2}>0$ such that for some continuous norm $\|\cdot\|$ on $S_{d}(\mathbb{R})$ one has

$$
\left|\left(S \Phi_{n}\right)(z \mathbf{f})\right| \leq K_{1} e^{K_{2}|z|^{2}\|\mathbf{f}\|^{2}}, \quad \forall z \in \mathbb{C}, \mathbf{f} \in S_{d}(\mathbb{R}), n \in \mathbb{N} .
$$

Then $\left(\Phi_{n}\right)_{n \in \mathbb{N}}$ converges strongly in $(S)^{*}$ to an unique Hida distribution.

Corollary 4 Let $(\Omega, \mathcal{B}, m)$ be a measure space and $\lambda \mapsto \Phi_{\lambda}$ be a mapping from $\Omega$ to $(S)^{*}$. We assume that the $S$-transform of $\Phi_{\lambda}$ fulfills the following two properties:

(i) The mapping $\lambda \mapsto\left(S \Phi_{\lambda}\right)$ (f) is measurable for every $\mathbf{f} \in S_{d}(\mathbb{R})$,

(ii) The $S \Phi_{\lambda}$ obeys a U-estimate

$$
\left|\left(S \Phi_{\lambda}\right)(z \mathbf{f})\right| \leq C_{1}(\lambda) e^{C_{2}(\lambda)|z|^{2}\|\mathbf{f}\|^{2}}, \quad z \in \mathbb{C}, \mathbf{f} \in S_{d}(\mathbb{R}),
$$

for some continuous norm $\|\cdot\|$ on $S_{d}(\mathbb{R})$ and for $C_{1} \in L^{1}(\Omega, m), C_{2} \in$ $L^{\infty}(\Omega, m)$.

Then

$$
\int_{\Omega} d m(\lambda) \Phi_{\lambda} \in(S)^{*}
$$

and

$$
S\left(\int_{\Omega} d m(\lambda) \Phi_{\lambda}\right)(\mathbf{f})=\int_{\Omega} d m(\lambda) S \Phi_{\lambda}(\mathbf{f}) .
$$




\section{Intersection local times}

Proposition 5 For $t \neq s$ the Bochner integral

$$
\delta\left(\mathbf{B}_{H}(t)-\mathbf{B}_{H}(s)\right):=\left(\frac{1}{2 \pi}\right)^{d} \int_{\mathbb{R}^{d}} d \lambda e^{i \lambda\left(\mathbf{B}_{H}(t)-\mathbf{B}_{H}(s)\right)}
$$

is a Hida distribution with S-transform given by

$$
S \delta\left(\mathbf{B}_{H}(t)-\mathbf{B}_{H}(s)\right)(\mathbf{f})=\left(\frac{1}{\sqrt{2 \pi}|t-s|^{H}}\right)^{d} e^{-\frac{1}{2|t-s|^{2 H}}\left|\int_{\mathbb{R}} d x \mathbf{f}(x)\left(M_{H} \mathbf{1}_{[s \wedge t, s \vee t]}\right)(x)\right|^{2}}
$$

for all $\mathbf{f} \in S_{d}(\mathbb{R})$.

Proof. The proof of this result follows from the application of Corollary 4 to the $S$-transform of the integrand function in (7) with respect to the Lebesgue measure on $\mathbb{R}^{d}$.

Since

$$
S e^{i \lambda\left(\mathbf{B}_{H}(t)-\mathbf{B}_{H}(s)\right)}(\mathbf{f})=e^{-\frac{|\lambda|^{2}}{2}(t-s)^{2 H}} e^{i \lambda \int_{\mathbb{R}} d x \mathbf{f}(x)\left(M_{H} 1_{[s, t]}\right)(x)},
$$

for $t>s$, and

$$
S e^{i \lambda\left(\mathbf{B}_{H}(t)-\mathbf{B}_{H}(s)\right)}(\mathbf{f})=e^{-\frac{|\lambda|^{2}}{2}(s-t)^{2 H}} e^{-i \lambda \int_{\mathbb{R}} d x \mathbf{f}(x)\left(M_{H} 1_{[t, s]}\right)(x)},
$$

for $t<s$ cf. e.g. HKPS93, clearly in both situations the measurability condition is fulfilled.

Independently of the particular case under consideration, we observe that for all $z \in \mathbb{C}$ and all $\mathbf{f} \in S_{d}(\mathbb{R})$ we find

$$
\begin{aligned}
\left|S e^{i \lambda\left(\mathbf{B}_{H}(t)-\mathbf{B}_{H}(s)\right)}(z \mathbf{f})\right| & =e^{-\frac{|\lambda|^{2}}{4}|t-s|^{2 H}}\left|e^{-\frac{|\lambda|^{2}}{4}|t-s|^{2 H} \pm i z \lambda \int_{\mathbb{R}} d x \mathbf{f}(x)\left(M_{H} 1_{[s \wedge t, s \vee t]}\right)(x)}\right| \\
& \leq e^{-\frac{|\lambda|^{2}}{4}|t-s|^{2 H}} e^{-\frac{|\lambda|^{2}}{4}|t-s|^{2 H}+|z||\lambda|\left|\int_{\mathbb{R}} d x \mathbf{f}(x)\left(M_{H} 1_{[s \wedge t, s \vee t]}\right)(x)\right|}
\end{aligned}
$$

where the latter exponential is bounded by

$$
e^{\frac{|z|^{2}}{|t-s|^{2 H}}\left|\int_{\mathbb{R}} d x \mathbf{f}(x)\left(M_{H} 1_{[s \wedge t, s \vee t]}\right)(x)\right|^{2}},
$$


because

$$
\begin{aligned}
& -\frac{|\lambda|^{2}}{4}|t-s|^{2 H}+|z||\lambda|\left|\int_{\mathbb{R}} d x \mathbf{f}(x)\left(M_{H} \mathbb{1}_{[s \wedge t, s \vee t]}\right)(x)\right| \\
= & -\left(\frac{|z|}{|t-s|^{H}}\left|\int_{\mathbb{R}} d x \mathbf{f}(x)\left(M_{H} \mathbb{1}_{[s \wedge t, s \vee t]}\right)(x)\right|-\frac{|\lambda|}{2}|t-s|^{H}\right)^{2} \\
& +\frac{|z|^{2}}{|t-s|^{2 H}}\left|\int_{\mathbb{R}} d x \mathbf{f}(x)\left(M_{H} \mathbb{1}_{[s \wedge t, s \vee t]}\right)(x)\right|^{2} .
\end{aligned}
$$

As a result,

$$
\left|S e^{i \lambda\left(\mathbf{B}_{H}(t)-\mathbf{B}_{H}(s)\right)}(z \mathbf{f})\right| \leq e^{-\frac{|\lambda|^{2}}{4}|t-s|^{2 H}} e^{\frac{|z|^{2}}{|t-s|^{2 H}}\left|\int_{\mathbb{R}} d x \mathbf{f}(x)\left(M_{H} 1_{[s \wedge t, s \vee t]}\right)(x)\right|^{2}},
$$

where, as a function of $\lambda$, the first factor is integrable on $\mathbb{R}^{d}$ and the second factor is constant.

An application of the result mentioned above completes the proof. In particular, it yields (8) by integrating (9), (10) over $\lambda$.

In order to proceed further the next result shows to be very useful. It improves the estimate obtained in [Ben03, Theorem 2.3] towards the characterization results stated in Corollaries 3 and 4.

Lemma 6 Let $H \in(0,1)$ and $f \in S_{1}(\mathbb{R})$ be given. There is a non-negative constant $C_{H}$ independent of $f$ such that

$$
\left|\int_{\mathbb{R}} d x f(x)\left(M_{H} \mathbb{1}_{[s, t]}\right)(x)\right| \leq C_{H}|t-s|\left(\sup _{x \in \mathbb{R}}|f(x)|+\sup _{x \in \mathbb{R}}\left|f^{\prime}(x)\right|+|f|\right)
$$

for all $s<t$.

Proof. Since $M_{H}$ and $M_{H}^{+}$are dual operators,

$$
\int_{\mathbb{R}} d x f(x)\left(M_{H} \mathbb{1}_{[s, t]}\right)(x)=\int_{s}^{t} d x\left(M_{H}^{+} f\right)(x),
$$

and thus

$$
\left|\int_{\mathbb{R}} d x f(x)\left(M_{H} \mathbb{1}_{[s, t]}\right)(x)\right| \leq|t-s| \sup _{x \in \mathbb{R}}\left|\left(M_{H}^{+} f\right)(x)\right|
$$


It has been shown in [Ben03, Proof of Theorem 2.3] that for $0<H<\frac{1}{2}$,

$$
\sup _{x \in \mathbb{R}}\left|\left(M_{H}^{+} f\right)(x)\right| \leq C_{H}^{\prime}\left(\frac{2}{\frac{1}{2}-H} \sup _{x \in \mathbb{R}}|f(x)|+\frac{1}{H+\frac{1}{2}} \sup _{x \in \mathbb{R}}\left|f^{\prime}(x)\right|\right),
$$

for some constant $C_{H}^{\prime}>0$ independent of $f$, while for $\frac{1}{2}<H<1$,

$$
\begin{aligned}
& \left|\left(M_{H}^{+} f\right)(x)\right| \\
\leq & C_{H}^{\prime \prime} \int_{\mathbb{R}} d y|f(y)||y-x|^{H-\frac{3}{2}} \\
= & C_{H}^{\prime \prime} \int_{|y-x|<1} d y|f(y)||y-x|^{H-\frac{3}{2}}+C_{H}^{\prime \prime} \int_{|y-x|>1} d y|f(y)||y-x|^{H-\frac{3}{2}} \\
\leq & C_{H}^{\prime \prime} \frac{2}{H-\frac{1}{2}} \sup _{x \in \mathbb{R}}|f(x)|+C_{H}^{\prime \prime} \int_{|y-x|>1} d y|f(y)||y-x|^{H-\frac{3}{2}}
\end{aligned}
$$

for some constant $C_{H}^{\prime \prime}>0$ also independent of $f$. Concerning the latter integral, observe that

$$
\begin{aligned}
\int_{|y-x|>1} d y|f(y)||y-x|^{H-\frac{3}{2}} & \leq|f|\left(\int_{|y-x|>1} d y|y-x|^{2 H-3}\right)^{1 / 2} \\
& =\frac{1}{\sqrt{1-H}}|f|
\end{aligned}
$$

leading to

$$
\sup _{x \in \mathbb{R}}\left|\left(M_{H}^{+} f\right)(x)\right| \leq C_{H}^{\prime \prime}\left(\frac{2}{H-\frac{1}{2}} \sup _{x \in \mathbb{R}}|f(x)|+\frac{1}{\sqrt{1-H}}|f|\right) .
$$

We are now ready to state the main result on intersection local times $L_{H}$ as well as on their subtracted counterparts $L_{H}^{(N)}$. For simplicity we shall use the notation

$$
\Delta:=\left\{\left(t_{1}, t_{2}\right) \in \mathbb{R}^{2}: 0<t_{1}<t_{2}<1\right\} .
$$

Theorem 7 For any $H \in(0,1)$ and for any pair of integer numbers $d \geq 1$ and $N \geq 0$ such that $2 N(H-1)+d H<1$, the Bochner integral

$$
L_{H}^{(N)}:=\int_{\Delta} d^{2} t \delta^{(N)}\left(\mathbf{B}_{H}\left(t_{2}\right)-\mathbf{B}_{H}\left(t_{1}\right)\right)
$$

is a Hida distribution. 
Proof. To prove this result we shall again use Corollary 4 with respect to the Lebesgue measure on $\Delta$. For this purpose let us denote the truncated exponential series by

$$
\exp _{N}(x):=\sum_{n=N}^{\infty} \frac{x^{n}}{n !} .
$$

It follows from (88) that the $S$-transform of $\delta^{(N)}$ is given by

$$
\begin{aligned}
& S\left(\delta^{(N)}\left(\mathbf{B}_{H}\left(t_{2}\right)-\mathbf{B}_{H}\left(t_{1}\right)\right)\right)(\mathbf{f}) \\
= & \left(\frac{1}{\sqrt{2 \pi}\left|t_{2}-t_{1}\right|^{H}}\right)^{d} \exp _{N}\left(-\frac{1}{2\left|t_{2}-t_{1}\right|^{2 H}}\left|\int_{\mathbb{R}} d x \mathbf{f}(x)\left(M_{H} \mathbb{1}_{\left[t_{1}, t_{2}\right]}\right)(x)\right|^{2}\right),
\end{aligned}
$$

which is a measurable function.

In order to check the boundedness condition, on $S_{d}(\mathbb{R})$ let us consider the norm $\|\cdot\|$ defined by

$$
\|\mathbf{f}\|:=\left(\sum_{i=1}^{d}\left(\sup _{x \in \mathbb{R}}\left|f_{i}(x)\right|+\sup _{x \in \mathbb{R}}\left|f_{i}^{\prime}(x)\right|+\left|f_{i}\right|\right)^{2}\right)^{\frac{1}{2}}, \quad \mathbf{f}=\left(f_{1}, \ldots, f_{d}\right) \in S_{d}(\mathbb{R}) .
$$

We observe that for $d=1$ this norm reduces to a continuous norm on $S_{1}(\mathbb{R})$,

$$
\|f\|=\sup _{x \in \mathbb{R}}|f(x)|+\sup _{x \in \mathbb{R}}\left|f^{\prime}(x)\right|+|f|, \quad f \in S_{1}(\mathbb{R}),
$$

which implies the continuity of the norm (12) for higher dimensions. By Lemma 6 we obtain

$$
\left|\int_{\mathbb{R}} d x \mathbf{f}(x)\left(M_{H} \mathbb{1}_{\left[t_{1}, t_{2}\right]}\right)(x)\right|^{2} \leq C_{H}^{2}\left|t_{2}-t_{1}\right|^{2}\|\mathbf{f}\|^{2},
$$

and thus

$$
\begin{aligned}
& \left|S\left(\delta^{(N)}\left(\mathbf{B}_{H}\left(t_{2}\right)-\mathbf{B}_{H}\left(t_{1}\right)\right)\right)(z \mathbf{f})\right| \\
\leq & \left(\frac{1}{\sqrt{2 \pi}\left|t_{2}-t_{1}\right|^{H}}\right)^{d} \exp _{N}\left(\frac{C_{H}^{2}}{2}|z|^{2}\left|t_{2}-t_{1}\right|^{2-2 H}\|\mathbf{f}\|^{2}\right),
\end{aligned}
$$

for all $z \in \mathbb{C}$ and all $\mathbf{f} \in S_{d}(\mathbb{R})$. Estimating the function $\exp _{N}$ by

$$
\exp _{N}\left(\frac{C_{H}^{2}}{2}|z|^{2}\left|t_{2}-t_{1}\right|^{2-2 H}\|\mathbf{f}\|^{2}\right) \leq\left|t_{2}-t_{1}\right|^{2 N(1-H)} e^{\frac{C_{H}^{2}}{2}|z|^{2}\|\mathbf{f}\|^{2}},
$$


we then obtain

$$
\left|S\left(\delta^{(N)}\left(\mathbf{B}_{H}\left(t_{2}\right)-\mathbf{B}_{H}\left(t_{1}\right)\right)\right)(z \mathbf{f})\right| \leq\left(\frac{1}{\sqrt{2 \pi}}\right)^{d}\left|t_{2}-t_{1}\right|^{2 N(1-H)-d H} e^{\frac{C_{H}^{2}}{2}|z|^{2}\|\mathbf{f}\|^{2}},
$$

where $\left|t_{2}-t_{1}\right|^{2 N(1-H)-d H}$ is integrable on $\Delta$ if, and only if, $2 N(1-H)-d H>$ -1 . The proof is completed by an application of Corollary 4 .

As a consequence, one may derive the chaos expansion for the (truncated) local times $L_{H}^{(N)}$.

Proposition 8 Given a $H \in(0,1)$ and a pair of integer numbers $d \geq 1$ and $N \geq 0$ such that $2 N(H-1)+d H<1$, the kernel functions $F_{H, \mathbf{n}}$ are given by

$F_{H, 2 \mathbf{n}}\left(u_{1}, \ldots, u_{2 n}\right)=\frac{1}{\mathbf{n} !}\left(\frac{1}{\sqrt{2 \pi}}\right)^{d}\left(-\frac{1}{2}\right)^{n} \int_{\Delta} d^{2} t \frac{1}{\left|t_{2}-t_{1}\right|^{2 H n+d H}} \prod_{i=1}^{2 n}\left(M_{H} \mathbb{1}_{\left[t_{1}, t_{2}\right]}\right)\left(u_{i}\right)$

for each $\mathbf{n} \in \mathbb{N}^{d}$ such that $n \geq N$. All other kernel functions $F_{H, \mathbf{n}}$ are identically equal to zero.

Proof. By Corollary 4 , the $S$-transform of the (truncated) local time $L_{H}^{(N)}$ is obtained by integrating (11) over $\Delta$. Hence, given a $\mathbf{f}=\left(f_{1}, \ldots, f_{d}\right) \in S_{d}(\mathbb{R})$,

$$
\begin{aligned}
S L_{H}^{(N)}(\mathbf{f})= & \left(\frac{1}{\sqrt{2 \pi}}\right)^{d} \int_{\Delta} d^{2} t \frac{1}{\left|t_{2}-t_{1}\right| d H} \sum_{n=N}^{\infty} \frac{(-1)^{n}}{2^{n}\left|t_{2}-t_{1}\right|^{2 H n}} \times \\
& \times \sum_{\substack{n_{1}, \cdots, n_{d} \\
n_{1}+\cdots+n_{d}=n}} \frac{1}{n_{1} ! \cdots n_{d} !} \prod_{j=1}^{d}\left(\int_{\mathbb{R}} d x f_{j}(x)\left(M_{H} \mathbb{1}_{\left[t_{1}, t_{2}\right]}\right)(x)\right)^{2 n_{j}} \\
= & \left(\frac{1}{\sqrt{2 \pi}}\right)^{d} \int_{\Delta} d^{2} t \sum_{n=N}^{\infty}\left(-\frac{1}{2}\right)^{n} \frac{1}{\left|t_{2}-t_{1}\right|^{2 H n+d H}} \times \\
& \times \sum_{\substack{n_{1}, \cdots, n_{d} \\
n_{1}+\cdots+n_{d}=n}} \frac{1}{\mathbf{n} !} \prod_{j=1}^{d}\left(\int_{\mathbb{R}} d x f_{j}(x)\left(M_{H} \mathbb{1}_{\left[t_{1}, t_{2}\right]}\right)(x)\right)^{2 n_{j}} .
\end{aligned}
$$

Comparing with the general form of the chaos expansion

$$
L_{H}^{(N)}=\sum_{\mathbf{n}}\left\langle: \omega^{\otimes \mathbf{n}}:, F_{H, \mathbf{n}}\right\rangle
$$


one concludes that

$F_{H, 2 \mathbf{n}}\left(u_{1}, \ldots, u_{2 n}\right)=\frac{1}{\mathbf{n} !}\left(\frac{1}{\sqrt{2 \pi}}\right)^{d}\left(-\frac{1}{2}\right)^{n} \int_{\Delta} d^{2} t \frac{1}{\left|t_{2}-t_{1}\right|^{2 H n+d H}} \prod_{i=1}^{2 n}\left(M_{H} \mathbb{1}_{\left[t_{1}, t_{2}\right]}\right)\left(u_{i}\right)$

for each $\mathbf{n} \in \mathbb{N}^{d}$ such that $n \geq N$, while all other kernels $F_{H, \mathbf{n}}$ vanish.

Theorem 7 shows that for $d=1$ all intersection local times $L_{H}$ are welldefined for all possible Hurst parameters $H$ in $(0,1)$. For $d \geq 2$, intersection local times are well-defined only for $H<1 / d$. Informally speaking, for $H \geq$ $1 / d$ with $d \geq 2$, the local times only become well-defined once subtracted the divergent terms. This "renormalization" procedure motivates the study of a regularization. As a computationally simple regularization we discuss

$$
L_{H, \varepsilon}:=\int_{\Delta} d^{2} t \delta_{\varepsilon}\left(\mathbf{B}_{H}\left(t_{2}\right)-\mathbf{B}_{H}\left(t_{1}\right)\right), \quad \varepsilon>0,
$$

where

$$
\delta_{\varepsilon}\left(\mathbf{B}_{H}\left(t_{2}\right)-\mathbf{B}_{H}\left(t_{1}\right)\right):=\left(\frac{1}{\sqrt{2 \pi \varepsilon}}\right)^{d} e^{-\frac{\left(\mathbf{B}_{H}\left(t_{2}\right)-\mathbf{B}_{H}\left(t_{1}\right)\right)^{2}}{2 \varepsilon}} .
$$

Theorem 9 Let $\varepsilon>0$ be given. For all $H \in(0,1)$ and all dimensions $d \geq 1$ the functional $L_{H, \varepsilon}$ is a Hida distribution with kernel functions given by

$$
\begin{aligned}
& F_{H, \varepsilon, 2 \mathbf{n}}\left(u_{1}, \ldots, u_{2 n}\right) \\
= & \frac{1}{\mathbf{n} !}\left(\frac{1}{\sqrt{2 \pi}}\right)^{d}\left(-\frac{1}{2}\right)^{n} \int_{\Delta} d^{2} t \frac{1}{\left(\varepsilon+\left|t_{2}-t_{1}\right|^{2 H}\right)^{n+\frac{d}{2}}} \prod_{i=1}^{2 n}\left(M_{H} \mathbb{1}_{\left[t_{1}, t_{2}\right]}\right)\left(u_{i}\right)
\end{aligned}
$$

for each $\mathbf{n}=\left(n_{1}, \ldots, n_{d}\right) \in \mathbb{N}^{d}$, and $F_{H, \varepsilon, \mathbf{n}} \equiv 0$ if at least one of the $n_{i}$ is an odd number. Moreover, if $2 N(H-1)+d H<1$, then when $\varepsilon$ goes to zero the (truncated) functional $L_{H, \varepsilon}^{(N)}$ converges strongly in $(S)^{*}$ to the (truncated) local time $L_{H}^{(N)}$.

Proof. As before, the first part of the proof follows from the Corollary 4 with respect to the Lebesgue measure on $\Delta$. By the definition of the $S$-transform, for all $\mathbf{f} \in S_{d}(\mathbb{R})$ one finds

$$
\begin{aligned}
& S \delta_{\varepsilon}\left(\mathbf{B}_{H}\left(t_{2}\right)-\mathbf{B}_{H}\left(t_{1}\right)\right)(\mathbf{f}) \\
= & \left(\frac{1}{\sqrt{2 \pi\left(\varepsilon+\left|t_{2}-t_{1}\right|^{2 H}\right)}}\right)^{d} e^{-\frac{1}{2\left(\varepsilon+\left|t_{2}-t_{1}\right|^{2 H}\right)}\left|\int_{\mathbb{R}} d x \mathbf{f}(x)\left(M_{H} 1_{\left[t_{1}, t_{2}\right]}\right)(x)\right|^{2}},
\end{aligned}
$$


which is measurable. Hence, by Lemma 6 , for all $z \in \mathbb{C}$ and all $\mathbf{f} \in S_{d}(\mathbb{R})$ one has

$\left|S \delta_{\varepsilon}\left(\mathbf{B}_{H}\left(t_{2}\right)-\mathbf{B}_{H}\left(t_{1}\right)\right)(z \mathbf{f})\right| \leq\left(\frac{1}{\sqrt{2 \pi\left(\varepsilon+\left|t_{2}-t_{1}\right|^{2 H}\right)}}\right)^{d} e^{C_{H}^{2}|z|^{2} \frac{\left|t_{2}-t_{1}\right|^{2}}{2\left(\varepsilon+\left|t_{2}-t_{1}\right|^{2 H}\right)}\|\mathbf{f}\|^{2}}$

with $\frac{\left|t_{2}-t_{1}\right|^{2}}{2\left(\varepsilon+\left|t_{2}-t_{1}\right|^{2 H}\right)}$ bounded on $\Delta$ and $\left(\varepsilon+\left|t_{2}-t_{1}\right|^{2 H}\right)^{-\frac{d}{2}}$ integrable on $\Delta$. By Corollary 4 , one may then conclude that $L_{H, \varepsilon} \in(S)^{*}$ and, moreover, for every $\mathbf{f}=\left(f_{1}, \ldots, f_{n}\right) \in S_{d}(\mathbb{R})$,

$$
\begin{aligned}
S L_{H, \varepsilon}(\mathbf{f})= & \int_{\Delta} d^{2} t S \delta_{\varepsilon}\left(\mathbf{B}_{H}\left(t_{2}\right)-\mathbf{B}_{H}\left(t_{1}\right)\right)(\mathbf{f}) \\
= & \left(\frac{1}{\sqrt{2 \pi}}\right)^{d} \int_{\Delta} d^{2} t \frac{1}{\left(\varepsilon+\left|t_{2}-t_{1}\right|^{2 H}\right)^{\frac{d}{2}}} \sum_{n=0}^{\infty} \frac{(-1)^{n}}{2^{n}\left(\varepsilon+\left|t_{2}-t_{1}\right|^{2 H}\right)^{n}} \times \\
& \times \sum_{\substack{n_{1}, \cdots, n_{d}=\\
n_{1}+\cdots+n_{d}=n}} \frac{1}{n_{1} ! \cdots n_{d} !} \prod_{j=1}^{d}\left(\int_{\mathbb{R}} d x f_{j}(x)\left(M_{H} \mathbb{1}_{\left[t_{1}, t_{2}\right]}\right)(x)\right)^{2 n_{j}} \\
= & \left(\frac{1}{\sqrt{2 \pi}}\right)^{d} \int_{\Delta} d^{2} t \sum_{n=0}^{\infty}\left(-\frac{1}{2}\right)^{n} \frac{1}{\left(\varepsilon+\left|t_{2}-t_{1}\right|^{2 H}\right)^{n+\frac{d}{2}}} \times \\
& \times \sum_{\substack{n_{1}, \cdots, n_{d} \\
n_{1}+\cdots+n_{d}=n}} \frac{1}{\mathbf{n} !} \prod_{j=1}^{d}\left(\int_{\mathbb{R}} d x f_{j}(x)\left(M_{H} \mathbb{1}_{\left[t_{1}, t_{2}\right]}\right)(x)\right)^{2 n_{j}} .
\end{aligned}
$$

As in the proof of Proposition 8 , it follows from the latter expression that the kernels $F_{H, \varepsilon, \mathbf{n}}$ appearing in the chaos expansion $L_{H, \varepsilon}=\sum_{\mathbf{n}}\left\langle: \omega^{\otimes \mathbf{n}}:, F_{H, \varepsilon, \mathbf{n}}\right\rangle$ vanish if at least one of the $n_{i}$ in $\mathbf{n}=\left(n_{1}, \ldots, n_{d}\right)$ is an odd number, otherwise they are given by

$$
\begin{aligned}
& F_{H, \varepsilon, 2 \mathbf{n}}\left(u_{1}, \ldots, u_{2 n}\right) \\
= & \frac{1}{\mathbf{n} !}\left(\frac{1}{\sqrt{2 \pi}}\right)^{d}\left(-\frac{1}{2}\right)^{n} \int_{\Delta} d^{2} t \frac{1}{\left(\varepsilon+\left|t_{2}-t_{1}\right|^{2 H}\right)^{n+\frac{d}{2}}} \prod_{i=1}^{2 n}\left(M_{H} \mathbb{1}_{\left[t_{1}, t_{2}\right]}\right)\left(u_{i}\right) .
\end{aligned}
$$

To complete the proof amounts to check the convergence. For this purpose we shall use Corollary 3, Since

$$
S L_{H, \varepsilon}^{(N)}(\mathbf{f})=\int_{\Delta} d^{2} t S \delta_{\varepsilon}^{(N)}\left(\mathbf{B}_{H}\left(t_{2}\right)-\mathbf{B}_{H}\left(t_{1}\right)\right)(\mathbf{f}),
$$


for all $z \in \mathbb{C}$ and all $\mathbf{f} \in S_{d}(\mathbb{R})$ we have

$$
\begin{aligned}
\left|S L_{H, \varepsilon}^{(N)}(z \mathbf{f})\right| & \leq \int_{\Delta} d^{2} t\left|S \delta_{\varepsilon}\left(\mathbf{B}_{H}\left(t_{2}\right)-\mathbf{B}_{H}\left(t_{1}\right)\right)(z \mathbf{f})\right| \\
& \leq\left(\frac{1}{\sqrt{2 \pi \varepsilon}}\right)^{d} \int_{\Delta} d^{2} t e^{\frac{C_{H}^{2}}{2 \varepsilon}|z|^{2}\left|t_{2}-t_{1}\right|^{2}\|\mathbf{f}\|^{2}} \leq\left(\frac{1}{\sqrt{2 \pi \varepsilon}}\right)^{d} e^{\frac{C_{H}^{2}}{2 \varepsilon}|z|^{2}\|\mathbf{f}\|^{2}}
\end{aligned}
$$

showing the boundedness condition. In addition, similar computations used to prove Theorem 7 yield for all $\left(t_{1}, t_{2}\right) \in \Delta$

$$
\begin{aligned}
& \left|S \delta_{\varepsilon}^{(N)}\left(\mathbf{B}_{H}\left(t_{2}\right)-\mathbf{B}_{H}\left(t_{1}\right)\right)(\mathbf{f})\right| \\
\leq & \left(\frac{1}{\sqrt{2 \pi}\left|t_{2}-t_{1}\right|^{H}}\right)^{d} \exp _{N}\left(\frac{C_{H}^{2}}{2}\left|t_{2}-t_{1}\right|^{2-2 H}\|\mathbf{f}\|^{2}\right) \\
\leq & \left(\frac{1}{\sqrt{2 \pi}}\right)^{d}\left|t_{2}-t_{1}\right|^{2 N(1-H)-d H} e^{\frac{C_{H}^{2}}{2}\|\mathbf{f}\|^{2}}
\end{aligned}
$$

which allows the use of the Lebesgue dominated convergence theorem to infer the other condition needed for the application of Corollary 3 ,

\section{Acknowledgments}

M.J.O. and J.L.S. would like to express their gratitude for the splendid hospitality of our colleagues and friends Victoria Bernido and Christopher Bernido during a very pleasant stay at Jagna during the $5^{\text {th }}$ Jagna International Workshop. Financial support of the FCT through POCI, PDCT, and PTDC projects is gratefully acknowledged.

\section{References}

[Ben03] C. Bender. An Itô formula for generalized functionals of a fractional Brownian motion with arbitrary Hurst parameter. Stochastic Processes Appl., 104:81-106, 2003.

[BK88] Yu. M. Berezansky and Yu. G. Kondratiev. Spectral Methods in Infinite-Dimensional Analysis. Naukova Dumka, Kiev, 1988. (in Russian). English translation, Kluwer Academic Publishers, Dordrecht, 1995. 
[dFDS00] M. de Faria, C. Drumond, and L. Streit. The renormalization of self-intersection local times. I. The chaos expansion. Infin. Dimens. Anal. Quantum Probab. Relat. Top., 3(2):223-236, 2000.

[dFHSW97] M. de Faria, T. Hida, L. Streit, and H. Watanabe. Intersection local times as generalized white noise functionals. Acta Appl. Math., 46:351-362, 1997.

[GRV03] M. Gradinaru, F. Russo, and P. Vallois. Generalized covariations, local time and Stratonovich Itô's formula for fractional Brownian motion with Hurst index $H \geq \frac{1}{4}$. Ann. Probab., 31(4):1772-1820, 2003.

[Hid75] T. Hida. Analysis of Brownian Functionals, volume 13 of Carleton Mathematical Lecture Notes. Carleton University, Ottawa, 1975.

[HKPS93] T. Hida, H. H. Kuo, J. Potthoff, and L. Streit. White Noise. An Infinite Dimensional Calculus. Kluwer Academic Publishers, Dordrecht, 1993.

[HN05] Y. Hu and D. Nualart. Renormalized self-intersection local time for fractional Brownian motion. Ann. Probab., 33:948-983, 2005.

[HN07] Y. Hu and D. Nualart. Regularity of renormalized selfintersection local time for fractional Brownian motion. Commun. Inf. Syst., 7(1):21-30, 2007.

[HØUZ96] H. Holden, B. Øksendal, J. Ubøe, and T. Zhang. Stochastic Partial Differential Equations: A Modeling, White Noise Functional Approach. Birkhäuser, Boston, Basel, and Berlin, 1996.

$\left[\mathrm{KLP}^{+} 96\right]$ Yu. G. Kondratiev, P. Leukert, J. Potthoff, L. Streit, and W. Westerkamp. Generalized functionals in Gaussian spaces: The characterization theorem revisited. J. Funct. Anal., 141:301-318, 1996.

[Kon75] Yu. G. Kondratiev. Spaces of Test and Generalized Functions of an Infinite Number of Variables. Master's thesis, University of Kiev, 1975. 
[KT80a] I. Kubo and S. Takenaka. Calculus on Gaussian white noise I. Proc. Japan Acad. Ser. A Math. Sci., 56:376-380, 1980.

[KT80b] I. Kubo and S. Takenaka. Calculus on Gaussian white noise II. Proc. Japan Acad. Ser. A Math. Sci., 56:411-416, 1980.

[Kuo96] H. H. Kuo. White Noise Distribution Theory. CRC Press, Boca Raton, New York, London, and Tokyo, 1996.

[NOL07] D. Nualart and S. Ortiz-Latorre. Intersection local time for two independent fractional Brownian motions. J. Theoret. Probab., 20(4):759-767, 2007.

[Oba94] N. Obata. White Noise Calculus and Fock Space, volume 1577 of LNM. Springer Verlag, Berlin, Heidelberg, and New York, 1994.

[PS91] J. Potthoff and L. Streit. A characterization of Hida distributions. J. Funct. Anal., 101:212-229, 1991.

[PT00] V. Pipiras and M. S. Taqqu. Integration questions related to fractional Brownian motion. Probab. Theory Related Fields, 118:251-291, 2000.

[Rez07] A. Rezgui. The renormalization of self intersection local times of fractional Brownian motion. Int. Math. Forum, 2(44):2161$2178,2007$.

[Ros87] J. Rosen. The intersection local time of fractional Brownian motion in the plane. J. Multivar. Anal., 23:37-46, 1987. 